\title{
On Times to Compute Shapes in 2D Tile Self-Assembly
}

\author{
Yuliy Baryshnikov $^{1}$ Ed Coffman ${ }^{2}$ Boonsit Yimwadsana ${ }^{2}$ \\ ${ }^{1}$ Bell Labs, Lucent Technologies, Murray Hill, NJ 07974 \\ ${ }^{2}$ Electrical Engineering Dept., Columbia University, New York, NY 10027
}

\begin{abstract}
We study the times to grow structures within the tile self-assembly model proposed by Winfree, and the possible shapes that can be achieved. Our earlier work was confined to the growth of rectangular structures, in which the rates of attachment of border tiles and rule tiles were the same. By varying the relative rates one can engineer interesting new shapes, which have been observed in the laboratory. We show that the results from an extension of our earlier stochastic models agree remarkably closely with experimental results. This is an important further demonstration of the validity and usefulness of our stochastic models, which has also been used to study error correction in DNA self assembly.
\end{abstract}

\section{The Tile Self-Assembly Model}

The general focus of the work here is on mathematical foundations of self assembly based on Winfrees DNA tile model [12] to be described shortly. More precisely, the emphasis is on the analysis of stochastic models. Although insightful such models and reference theories are ubiquitous in the physical sciences, they remain a fertile ground for self-assembly research in DNA-Based Computing, where stochastic analysis has only recently begun. The early work of Adleman [3] and colleagues and that of the authors $[5,4,6]$ sets the stage in this area, and serves as the point of departure for the analysis here.

The seminal mathematical tile model of DNA self-assembly, as developed by Winfree [12] and pursued by many others, has led to a much improved understanding of DNA self-assembly in two dimensions. At the physical layer being modeled, single-strand DNA molecules are manipulated to form DNA molecules (e.g., double-crossover molecules [7, 8]) which are designed to assemble (bond) with other such molecules in a two dimensional crystal-growth process obeying bonding rules determined by the molecular motifs [12]. These building-block molecules are modeled as tiles.

There are three types of tiles involved in a self-assembly process: rule tiles, border tiles, and seed tiles. They participate in a growth process beginning at the origin of the positive lattice; the unit squares of the lattice are the potential sites occupied by tiles.

- The seed tile occupies the lower-left corner of the positive lattice and is responsible for initiating the tile self-assembly process. Only border tiles, as described next, can stick to the two free (upper and right-hand) sides of the seed tiles.

- A border tile can join the structure only by attaching to the seed tile or another border tile along the horizontal and vertical boundaries of the positive lattice, each such attachment extending one of the borders of the structure assembled so far. 
- A rule tile can attach to the growing structure at any available site which is adjacent to occupied sites both to the left and below the available site, where either site may contain a border tile.

In the probability model governing growth (cf. $[4,6]$ ), as soon as a site becomes available to a rule tile (for attachment to the left and below) its waiting time for such a tile is exponentially distributed with mean 1. All such waiting times are independent. The attachment of new border tiles is also subject to independent exponential waiting times, but with a different rate parameter $\alpha$. In the analysis of the first such model [4], the border (input) tiles were assumed to be prefabricated, occupying sites, say, from $(0,0)$ to $(\mathrm{M}, 0)$ and to $(0, \mathrm{~N})$, the so-called $\mathrm{L}$ input; the problem was to estimate the time $C_{M, N}$ until a rule tile became attached to the site at $(\mathrm{M}, \mathrm{N})$.

One of the principal tools used in solving this type of problem has been the analysis of the TASEP (Totally Asymmetric Simple Exclusion Process) [9]; the main result is given below in terms of fluid limits where the discrete position variables $\mathrm{i}$ and $\mathrm{j}$ are replaced by continuous variables $\mathrm{x}$ and $\mathrm{y}$. We have, with the obvious change in notation,

$$
\lim _{(x, y) \rightarrow(\infty, \infty)} C_{(x, y)}=\sup _{\gamma}(\sqrt{x}+\sqrt{y})^{2}
$$

where $\gamma$ represents all possible paths from the origin (the seed tile) to the point $(x, y)$. In other words, the time it takes to place a tile at position $(x, y)$ is the maximum time it takes to place tiles along all possible path from the origin to position $(x, y)$. In [4], we show that the path is the straight line connecting the origin, the location of the seed tile, to point $(x, y)$ in the fluid limit case.

In the more general setting, where border tiles are not prefabricated, but take part in the self-assembly process, the problem becomes much more interesting and entails the assembly of a much larger class of shapes, particularly when $\alpha<1$, i.e., the border-tile attachment rate is less than that of the rule tiles. ${ }^{1}$ The more general extremal problem is illustrated in Figure 1. Since $\alpha<1$; a path along which the sum of expected attachment times is maximum must first move along one of the axes; the slow growth rate of the borders is less than the rate of tiling the rectangles bounded by the borders. At a point where these rates balance, the trajectory is determined by the rectangle tiling result of (1).

In Figure 1(b), the dotted line shows the shape of self-assembled structure before the border reaches point $K$ during the process. When the crystal boundary is below point $K$, the rule tiles can fill in the square covered by the length of the border before a new border tile arrive since the border tiles' interarrival times are much greater than the time that the rule tiles fill in the square when the edge of the square is small. This means the time it takes to place a tile depends on the speed of the border tile attachment. When the boundary grows beyond point $K$, the rule tiles cannot complete the square in time before the next arrival of a border tile, hence the attachment of rule tiles contribute to the speed of the self-assembly process.

This argument holds true for the case where $x \geq y$ and $x \leq y$. Thus, in the figure, to compute a path to $(x, y)$ along which expected total attachment time is maximum, one must find this maximum among paths like $\overline{b a}$; if $x \leq y$, and $\overline{d c}$, if $x \geq y$.

With $z$ as defined in the figure, the extremal path of tile attachments will start from the origin, go to position $(0, y-z)$ in expected time $(y-z) / \alpha$ for $x \leq y$ or $(x-z, 0)$ in expected time $(x-z) \alpha$ for

\footnotetext{
${ }^{1} \mathrm{~K}$. Fujibayashi working in E. Winfrees lab at $\mathrm{Cal}$ Tech notes that $\alpha \approx 1 / 2$ was common in certain of his experiments, where $\alpha$ is a stoichiometric parameter giving the concentration of border tiles. In this case he found that the profile of the self-assembly structure is triangular, a fact that will also emerge in our purely mathematical framework.
} 


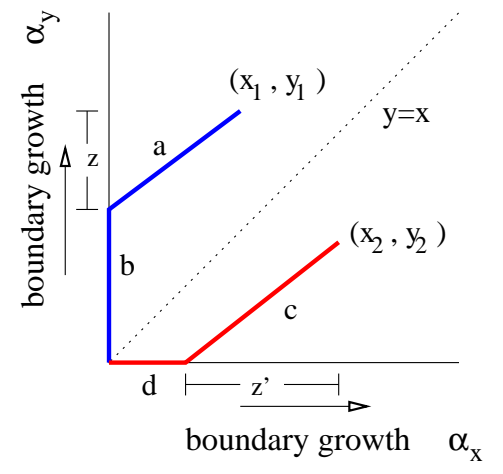

(a)

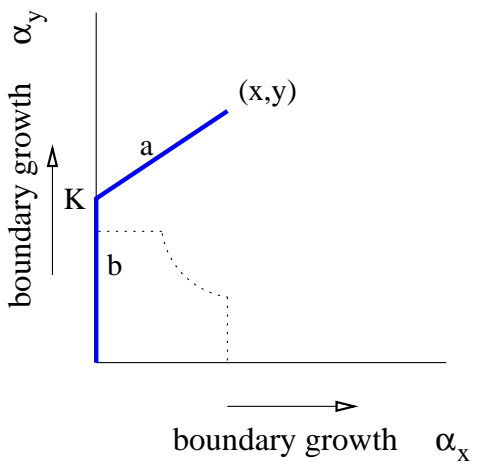

(b)

Figure 1: (a) The paths that contribute to the time required for a tile to attach to the position $(x, y)$. The path $\overline{b a}$ is followed when $x<y$ and the path $\overline{d c}$ is followed when $x>y$. (b) The dotted line illustrates the boundary of the shape of self-assembled structure before the border tiles reach point $K$ during the process. When the crystal boundary is below point $K$, the rule tiles (in the fluid limit) fill in the square covered by the length of the border before a new border tile arrives. Beyond point $K$, the rule tiles cannot complete the square before the next arrival of a border tile, so the attachment of rule tiles determines the speed of the approach to $x, y$ ).

$x \geq y$, and then go on to reach $(x, y)$. We need only find the supremum over $z$ to identify the extremal path. Thus, for finding the time to reach point $(x, y)$ where $x \geq y$,

$$
C_{x, y} \sim \sup _{z}\left((\sqrt{x}+\sqrt{z})^{2}+\frac{y-z}{\alpha}\right)
$$

as $(x, y) \rightarrow(\infty, \infty)$. A calculation for $C_{x, y}$ then shows that

$$
\begin{aligned}
C_{x, y} & \sim x\left(1+\frac{\alpha}{1-\alpha}\right)^{2}+\frac{y}{\alpha}-\frac{x \alpha}{(1-\alpha)^{2}} \\
& =\frac{y}{\alpha}+\frac{x}{1-\alpha}
\end{aligned}
$$

as $(x, y) \rightarrow(\infty, \infty)$, where $z=x\left(\frac{\alpha}{\alpha-1}\right)^{2}, x<y$ and $z<y$.

An analysis of the alternate path $\overline{c d}$ for $x \geq y$ follows the same arguments and yields the same result with $x$ and $y$ interchanged. By fixing the computing time $C_{x, y}$ (e.g. at an instant of time), we can plot values of $x$ and $y$ for different values of $\alpha$ as shown in Figure 2 .

For $0 \leq \alpha<1 / 2$, the shape of the self-assembled crystal looks like the one shown in Figure 2(a). When $\alpha=1 / 2$, the shape of the self-assembled crystal is triangular as shown in Figure 2. This corresponds to the shape suggested by K. Fujibayashi's simulation. When $1 / 2<\alpha<1$, the shape of the structures has an L-shape with sharp corners as shown in Figure 2(b). Then when $\alpha \geq 1$, the shape of the selfassembled structure is no longer linear like, and it is described by the equation suggested in [4] which analyzed self-assembly systems whose rate of growth for the border tiles is greater than or equal to the rate of growth for the rule tiles. 


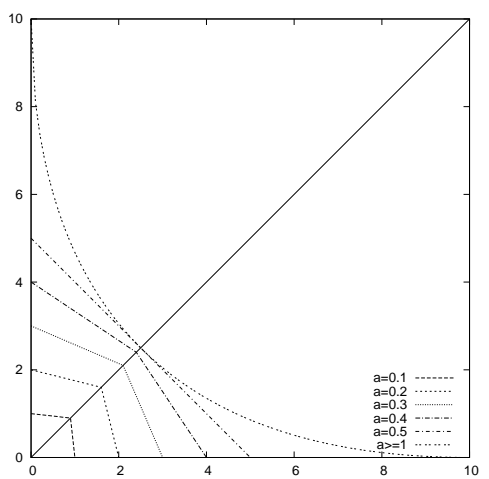

(a) $\alpha \leq 0.6$

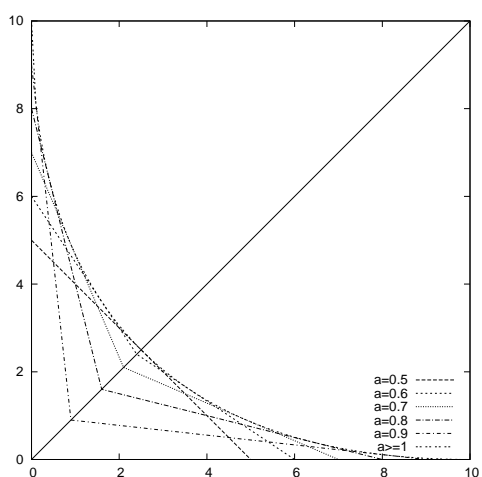

(b) $\alpha \geq 0.5$

Figure 2: The shape of tile self-assembly for different values of border growth $\alpha$.

It is clear from (3) that the time a tile takes to attach to position $(x, y)$ depends linearly on the value of $x$ and $y$, given that $\alpha$ is a constant. This explains why the asymptotic shape of the structure looks like simple geometric shapes, especially when $\alpha$ is approximately $1 / 2$ the shape becomes triangular.

Remarkably, the simulations of self-assembly structures agree with our prediction for all values of $\alpha \leq \frac{1}{2}$. Figure 3 shows the simulation results for various values of $\alpha$.

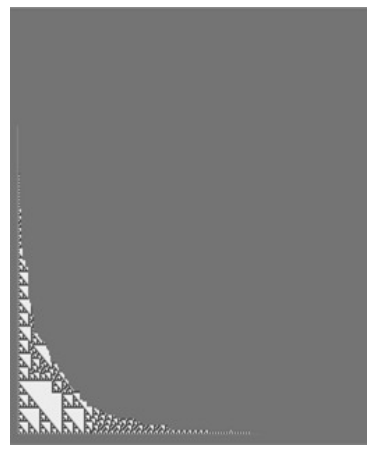

(a) $\alpha=1.2$

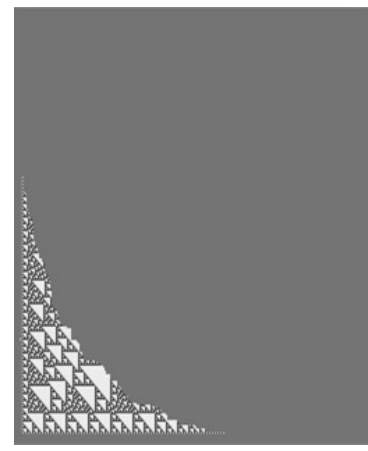

(b) $\alpha=0.8$

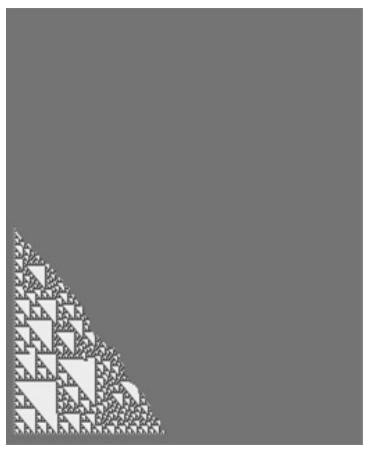

(c) $\alpha=0.5$

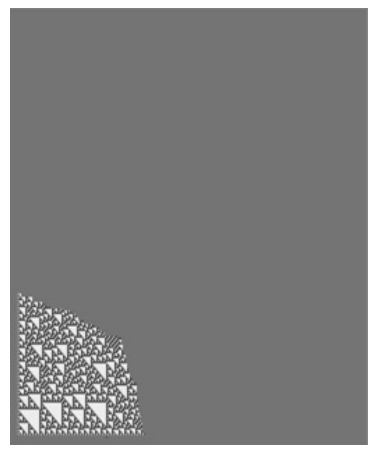

(d) $\alpha=0.3$

Figure 3: Simulation of 2D structures with various values of $\alpha$

It is possible to extend the scope of our discussion to be even more general by allowing the rates of border growth to differ on the vertical and horizontal axes.

\section{Different Rates of Border Growth}

Let $\alpha_{1}$ be the ratio of the attachment rate of the vertical border tiles to the rule-tile attachment rate, and let $\alpha_{2}$ be the ratio of the attachment rate of the horizontal border tiles to that of the rule tiles. The paths that determine the time taken by a tile to arrive at position $(x, y)$ with $x<y$ (respectively $x>y$ ) do not 
depend on the border tiles that are growing in the horizontal (respectively vertical) direction. We arrive at

$$
\begin{aligned}
C_{x, y} & =\frac{y}{\alpha_{1}}+\frac{x}{1-\alpha_{1}} & & \text { for } x<y \\
C_{x, y} & =\frac{y}{1-\alpha_{2}}+\frac{x}{\alpha_{2}} & & \text { for } x>y
\end{aligned}
$$

as $(x, y) \rightarrow(\infty, \infty)$. Figure 4 gives an example of the shape of self-assembly structures with different border growth rates.

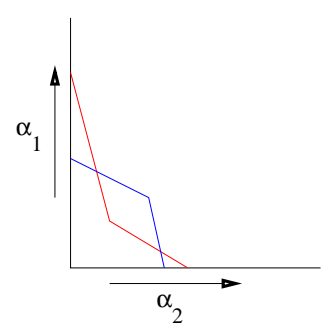

Figure 4: Simulation of 2D structures with different border growth rates. The shapes are no longer symmetric along $y=x$.

Crystal self-assembly is typically designed to grow indefinitely yielding congruent shapes at different times. A problem arises when we want a specific finite shape. Although we can design tile sets so that the growth process will stop at some pre-specified point, the complexity of the tile sets is high (cf. $[2,1,10,11])$. In order to create shapes that are useful and economically efficient, a small number of simple tiles is desirable. A straightforward method of creating desired shapes is simply to remove the supply of tiles completely when we know that the desired crystal size has been reached. This method works since the shape (fluid limit) approximation above allows one to estimate the size of the structure and the time it takes to create a specific structure.

\section{Conclusions}

As is well known, essentially arbitrary 2D shapes can be created by tile self assembly (cf. e.g., [11, 12]). For example, one technique is to create a domino pattern that serves as a skeleton on which to grow some given shape; the length $L$ of a leg of the pattern can be determined by a counter structure of order $\log L$ width. Figure 5(a) shows an example which begins with a (blue) seed from which growth proceeds in each of the four directions. At the ends of legs new (red) seed structures can self-assemble to give the domino branching patterns. Once the domino pattern is in place, or as it self assembles, the growth as described earlier can flesh out the desired shape. The domino patterns define independent, elementary growth regions as illustrated in Figure 5(a). In simple cases, expected times to compute such shapes may be expressible as the expected maximum of the times to grow the independent regions (cf. [4]). But in general, the computations of overall expected growth times are complex.

Acknowledgement We are grateful to K. Fujibayashi for pointing out and discussing the problem of (slowly) self assembling border tiles. 


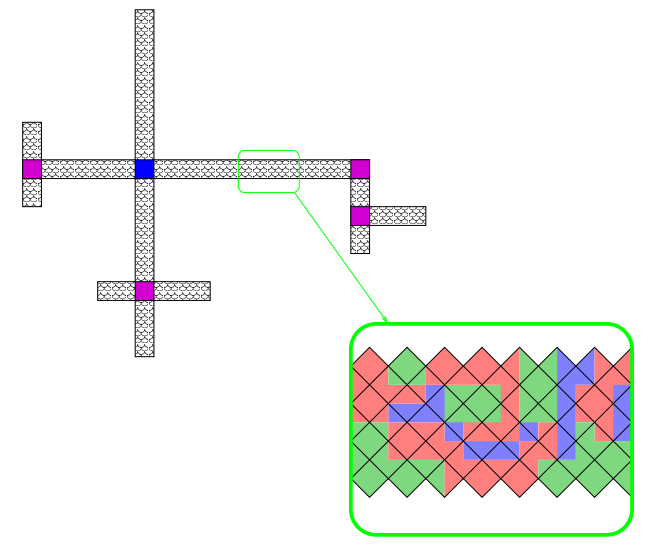

(a) skeleton

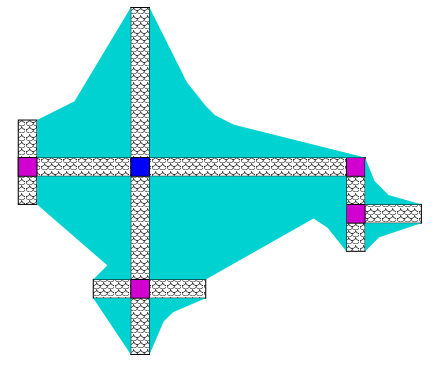

(b) body

Figure 5: The shape of tile self-assembly model built on a predesigned skeleton.

\section{References}

[1] L. Adleman, Q. Cheng, A. Goel, and M.-D. Huang. Running time and program size for self-assembled squares. In Proc. ACM Symp. Th. Comput., pages 740-748, 2001.

[2] L. Adleman, Q. Cheng, A. Goel, M.-D. Huang, D. Kempe, P. Moisset de Espanés, and P. Rothemund. Combinatorial optimization problems in self-assembly. In Proc. ACM Symp. Th. Comput., pages 23-32, Montreal, Canada, 2002.

[3] L. Adleman, Q. Cheng, A. Goel, M.-D. Huang, and H. Wasserman. Linear self-assemblies: Equilibria, entropy, and convergence rates. In Elaydi, Ladas, and Aulbach, editors, New progress in difference equations. Taylor and Francis, London, 2004.

[4] Y. Baryshnikov, E. Coffman, and P. Momčilović. DNA-based computation times. In Proc. of the Tenth International Meeting on DNA Computing, Milan, Italy, June 2004.

[5] Y. Baryshnikov, E. Coffman, and P. Momčilović. Incremental self-assembly in the fluid limit. In Proc. 38th Ann. Conf. Inf. Sys. Sci., Princeton, NJ, 2004.

[6] Y. Baryshnikov, E. Coffman, N. Seeman, and B. Yimwadsana. Self correcting self assembly: Growth models and the hammersley process. In Proc. of the Eleventh International Meeting on DNA Computing, London, Ontario, 2005.

[7] B. Ding, R. Sha, and N. Seeman. Pseudohexagonal 2D DNA crystals from double crossover cohesion. J. Am. Chem. Soc., 126:10230-10231, 2004.

[8] T.-J. Fu and N. Seeman. DNA double crossover structures. Biochemistry, 32:3211-3220, 1993.

[9] T. M. Liggett. Interacting Particle Systems. Springer-Verlag, New York, 1985.

[10] P. Rothemund and E. Winfree. The program-size complexity of self-assembled squares. In Proc. ACM Symp. Th. Comput., pages 459-468, 2001.

[11] David Soloveichik and Erik Winfree. Complexity of self-assembled shapes. In Proc. of the Tenth International Meeting on DNA Computing, 2004.

[12] E. Winfree. Algorithmic Self-Assembly of DNA. PhD thesis, California Institute of Technology, Pasadena, CA, 1998. 